\title{
Introducing IES
}

\author{
George J. Davies - Terry R. Malone - Kent E. Timm
}

It is with great excitement and pride that we introduce the inaugural issue of Isokinetics and Exercise Science (IES).

There is a time for all things! The time has come for a journal dedicated to the science of isokinetics and its clinical applications.

Because of the dramatic increase in and the proliferation of interest, utilization, and publications in this area over the last decade, the need has arisen to have a specialized journal to share and disseminate professional information in the field of isokinetics. IES is our timely response to this challenge.

IES meets the professional needs of the contemporary exercise scientist, medical practitioner, allied health clinician, and industrial specialist through a consolidating focus on the field of isokinetics and serves as a vehicle to unify theoretical, physiological, and clinical information into one source.

This comprehensive approach includes traditional articles on basic and applied isokinetic research, as well as practical features incorporating isokinetic technology and techniques into everday clinical situations. This unique balance of the scientific and practical addresses needs of the practicing physician, surgeon, physical therapist, athletic trainer, chiropractor, exercise physiologist, industrial specialist, and occupational therapist as each attempts to meet the exercise needs of clients and patients. IES promises to become the focal journal for this exciting, ever-expanding field.

The journal will include the following departments: feature articles, literature review articles, clinical research articles, laboratory research articles, case studies, specific pathology protocols, creative clinical applications (how I treat), clinical research abstracts, student corner, product news, and other topics of professional interest.

We invite you to join us in this exciting new opportunity to learn more about isokinetics and exercise science! IES 